\title{
Nonlinear mode decomposition: a new algorithm of interest for the biomedical field?
}

\author{
Humeau-Heurtier A* \\ University of Angers, LARIS - Laboratoire Angevin de Recherche en Ingénierie des Systèmes, 62 avenue Notre-Dame du Lac, 49000 Angers, France
}

The empirical mode decomposition (EMD) has been proposed by Huang et al. to adaptively decompose a signal into a few intrinsic mode functions (IMFs) that are zero-mean AM-FM components [1]. Since its introduction in 1998, EMD has been used in a variety of fields. Nevertheless, EMD suffers from some drawbacks as pointed out by many authors (see, e.g., [2]). This is why other EMD-based approaches have emerged, as [2-6] to cite only a few.

Recently, the nonlinear mode decomposition (NMD) has been proposed as another adaptive algorithm to decompose a signal into oscillations, simultaneously removing the noise [7]. From its performances on synthetic and real biomedical data, NMD has shown to overperform other methods [7].

NMD is based on a combination of a time-frequency analysis [8], surrogate data tests, and a harmonic identification [9]. More precisely, the algorithm relies on four steps [7]: (i) the extraction of the fundamental harmonic of an NM from the signal time-frequency representation [8]; (ii) the search of all its possible harmonics; (iii) the identification of the true harmonics; (iv) the reconstruction of the full NM from the summation of the true harmonics; the subtraction of this NM from the signal; this is iterated on the residual till a stopping criterion is met.

In its cornerstone paper, NMD has been applied on simulated signals as well as on real life data [7]. For the latter case, laser Doppler flowmetry (LDF) signals that reflect microvascular blood flow have been processed [7]. The results revealed that NMD is able to decompose data into oscillations linked with physiological activities. NMD has also shown success in removing cardiac artifacts from electroencephalogram signals [7].

Copyright: (C2015 Humeau-Heurtier A, Bianciardi G. This is an open-access article distributed under the terms of the Creative Commons Attribution License, which permits unrestricted use, distribution, and reproduction in any medium, provided the original author and source are credited.
From its performances, NMD can become of great interest for the biomedical field. Many applications are expected to emerge in medicine and biology. Moreover, the code for running NMD can be downloaded for free (http://www.physics.lancs.ac.uk/research/nbmphysics/diats/ $\mathrm{nmd} /)$.

\section{References}

1. Huang NE, Shen Z, Long SR, Wu MC, Shih HH, et al. (1998) The empirical mode decomposition and the Hilbert spectrum for nonlinear and non-stationary time series analysis. Proc R Soc London A 454: 903-995.

2. Wu Z, Huang NE (2009) Ensemble empirical mode decomposition: a noise-assisted data analysis method. Adv Adapt Data Anal 1 : 1-41.

3. Yeh JR, Shieh JS (2010) Complementary ensemble empirical mode decomposition: a novel noise enhanced data analysis method. Adv Adapt Data Anal 2: 135-156.

4. El'hadi Bekka R, Berrouche Y (2013) Improvement of ensemble empirical mode decomposition by over-sampling. Adv Adapt Data Anal 5. 1350012

5. Colominas MA, Schlotthauer G, Torres ME (2014) Improved complete ensemble EMD: A suitable tool for biomedical signal processing. Biomed Signal Process Control 14: $19-29$.

6. Xue X, Zhou J, Xu Y, Zhu W, Li C (2015) An adaptively fast ensemble empirical mode decomposition method and its applications to rolling element bearing fault diagnosis. Mech Syst Signal Process 62-63: 444-459.

7. Iatsenko D, McClintock PVE, Stefanovska A (2015) Nonlinear mode decomposition: A noise-robust, adaptive decomposition method. Phys Rev E Stat Nonlin Soft Matter Phys 92: 032916. [Crossref]

8. Iatsenko D, McClintock PVE, Stefanovska A (2015) Linear and synchrosqueezed time-frequency representations revisited: Overview, standards of use, resolution, reconstruction, concentration, and algorithms. Digit Signal Process 42: 1-26.

9. Sheppard LW, Stefanovska A, McClintock PVE (2011) Detecting the harmonics of oscillations with time-variable frequencies. Phys Rev E Stat Nonlin Soft Matter Phys 83: 016206. [Crossref]
Correspondence to: Humeau-Heurtier A, University of Angers, LARIS Laboratoire Angevin de Recherche en Ingénierie des Systèmes, 62 avenue NotreDame du Lac, 49000 Angers, France, E-mail: anne.humeau@univ-angers.fr

Received: September 01, 2015; Accepted: September 10, 2015; Published: September 15, 2015 